\title{
Gastric cardiac carcinomas involving the esophagus are more adequately staged as gastric cancers by the 7th edition of the American Joint Commission on Cancer Staging System
}

\author{
Qin Huang ${ }^{1,2,3}$, Jiong Shi ${ }^{1}$, Anning Feng ${ }^{1}$, Xiangshan Fan ${ }^{1}$, Lihua Zhang ${ }^{1}$,
} Hiroshi Mashimo ${ }^{3,4}$, Daniel Cohen ${ }^{3,5}$ and Gregory Lauwers ${ }^{3,6}$

${ }^{1}$ Department of Pathology of the Nanjing Drum Tower Hospital, Nanjing, China; ${ }^{2}$ Department of Pathology and Laboratory Medicine, West Roxbury, MA, USA; ${ }^{3}$ Harvard Medical School, West Roxbury, MA, USA; ${ }^{4}$ Department of Gastroenterology, West Roxbury, MA, USA; ${ }^{5}$ Department of Thoracic Surgery of Veterans Affairs Boston Healthcare System, West Roxbury, MA, USA and ${ }^{6}$ Department of Pathology of Massachusetts General Hospital, West Roxbury, MA, USA

The aim of this study was to compare the 7th with the 6th edition of the American Joint Commission on Cancer Staging System for prognostic stratification of gastric cardiac carcinomas involving the esophagus. We retrospectively compared differences in pathological stages with patient survival between the 7th and the 6th staging systems in 142 consecutive resection cases of this cancer. Patient median age was 65 years. The male-female ratio was 3.3. The epicenter of all tumors was within $5 \mathrm{~cm}$ below the gastroesophageal junction. The median tumor size was $5.0 \mathrm{~cm}$. Most tumors $(79 \%)$ were typical adenocarcinomas and the rest showed uncommon histology types. Using the guidelines for gastric cancer, this group of cancer was better stratified by the 7th than the 6th edition of the staging system, especially for pathological nodal $(\mathrm{pN})$ and overall stage pllIC. Patients with celiac axis nodal disease had the 5-year survival rate worse than those staged at pN3A and pllIA. Patients staged at PT3 and pN3B had the 5-year survival rate worse than those at pM1 and pIV. We showed that the overall stage of gastric cardiac carcinomas was better stratified by gastric than by esophageal cancer grouping. We conclude that these tumors are better stratified with the 7th than the 6th edition of the gastric staging system, especially for plll cancers, and better staged by the new gastric than esophageal cancer staging system. We propose that the staging of these tumors be reverted to gastric grouping and combine pT3 and pN3B into the overall stage pIV.

Modern Pathology (2011) 24, 138-146; doi:10.1038/modpathol.2010.183; published online 17 September 2010

Keywords: cancer; esophagus; gastroesophageal junction; staging; stomach

Gastric cardiac carcinomas involving the esophagus were traditionally staged as gastric cancers, accord-

Correspondence: Dr Q Huang, MD, PhD, Department of Pathology and Laboratory Medicine, Veterans Affairs Boston Healthcare System and Harvard Medical School, 1400 VFW Parkway, West Roxbury, MA 02132, USA.

E-mail: qinhuang2005@gmail.com

The major findings of this study were presented at the 2010 Digestive Disease Week annual meeting in New Orleans, Louisiana, USA, and published in an abstract form in Gastroenterology.

Received 14 May 2010; revised 6 August 2010; accepted 12 August 2010; published online 17 September 2010 ing to the 6th edition of the American Joint Commission on Cancer (AJCC 6th) Staging System. ${ }^{1}$ However, this practice has been challenged for its prognostic value. ${ }^{2-4}$ Furthermore, the AJCC 6th staging system was deemed to be flawed with regard to the $\mathrm{pN}$ staging system and also inaccurate in predicting patient outcomes after therapy. ${ }^{5-7}$ To address these challenges, a modified staging scheme was offered in the 7th edition of the AJCC staging system (AJCC 7th), which was published recently. ${ }^{8}$ However, the usefulness and validity of this new system for predicting survival of patients with gastric cancer has not been independently 
evaluated, especially with regard to the $\mathrm{pN}$ staging system. In addition, the new AJCC 7th staging system requires gastric cardiac carcinomas involving the esophagus to be staged with the esophageal, rather than the gastric, cancer staging scheme. ${ }^{8}$ The rationale and strength of this guideline has not been adequately assessed independently. Therefore, the aim of this study was to compare and contrast outcomes predicted by the AJCC 7th in comparison with the AJCC 6th system, in patients with surgically resected gastric cardiac carcinomas involving the esophagus at a single high-volume tertiary medical center in Nanjing, China.

\section{Materials and methods}

\section{Selection of Patients}

In this retrospective validation study on gastric cancer staging, we searched computerized pathology files stored in the Department of Pathology of the Nanjing Drum Tower Hospital in Nanjing, China, over the period from May 2005 through to October 2009 for consecutive resection cases $(n=172)$ of histopathologically confirmed carcinomas centered in the proximal stomach. Each pathology report was reviewed along with tumor gross images, if available, for tumor epicenter location, tumor-nodemetastasis (TNM) information, the presence or absence of distal esophageal involvement, and histopathological types of carcinomas. Our inclusion criteria were: (1) tumors with epicenters within $5 \mathrm{~cm}$ below the gastroesophageal junction and (2) tumor involvement of the distal esophagus. Exclusion criteria were: (1) chemoradiation therapy before resection surgery and (2) absence of follow-up information. As a result, 142 cases were eligible for the study (Table 1). Clinical follow-up information was acquired through telephone interviews of patients or their family members. The study protocol was approved by the Medical Ethics Committee of the Nanjing Drum Tower Hospital in Nanjing, China.

\section{Surgery}

The choice of operative approach for each tumor was based on tumor location, extent, depth of invasion, and functional status of patients (Table 1). The majority of cases underwent either a subtotal proximal gastrectomy with transhiatal resection of the distal esophagus or a total gastrectomy with transhiatal resection of the distal esophagus. A minority of patients had distal esophagectomy with proximal gastrectomy through a thoracotomy (Table 1). After resection, the specimen was opened and the relationship between the tumor epicenter and the gastroesophageal junction was determined. The gastroesophageal junction was defined as the most proximal end of gastric longitudinal mucosal folds. All cases showed tumor epicenters located within $5 \mathrm{~cm}$ below the gastroesophageal junction. Lymphadenectomy was carried out routinely in the paragastric region for pathological determination of the status of regional nodal metastasis. Specific remote location of lymph nodes was dissected and the nodes were submitted by surgeons for microscopic examination. Because of the retrospective nature of the study, specific distant lymphadenectomy was not standardized or performed in every case.

\section{TNM Staging}

Pathology reports were reviewed, and routine histology slides of each case were retrieved and evaluated. The tumor AJCC staging statement described in the report was confirmed or modified upon review of histology slides. On average, $10( \pm 5)$ slides per case were reevaluated. We used the staging terminology defined by both AJCC 7th and AJCC 6th systems to stage our series of gastric cardiac carcinomas involving the esophagus as gastric, and also as esophageal, cancers.

Depth of tumor invasion was determined as pathological tumor (pT) stage, with the deepest point of invasion into the gastric wall recorded as a representative pT stage. The overall number of lymph nodes included any positive plus all

Table 1 Patient demographic and tumor clinicopathological characteristics

\begin{tabular}{|c|c|c|c|}
\hline Characteristics & Type & Number & $\%$ \\
\hline \multirow[t]{2}{*}{ Age (years) } & Median & 65 & \\
\hline & Range & $47-90$ & \\
\hline \multirow{2}{*}{ Gender } & Male/female & $109 / 33$ & \\
\hline & $\mathrm{M}: \mathrm{F}$ ratio & & 3.3 \\
\hline \multirow[t]{3}{*}{ Surgery } & Ivor-Lewis operation & 10 & 7 \\
\hline & Total gastrectomy & 22 & 15 \\
\hline & Partial gastrectomy & 110 & 77 \\
\hline \multirow[t]{2}{*}{ Tumor size $(\mathrm{cm})$} & Median & 5 & \\
\hline & Range & $0.5-12$ & \\
\hline \multirow[t]{7}{*}{ Histopathology } & Adenocarcinoma, NOS & 112 & 79 \\
\hline & Carcinoma with micropapillary feature & 24 & 17 \\
\hline & Adenosquamous carcinoma & 9 & 6 \\
\hline & Mucinous carcinoma & 9 & 6 \\
\hline & Signet ring cell carcinoma & 23 & 16 \\
\hline & Carcinoma with neuroendocrine feature & 7 & 5 \\
\hline & Carcinoma with mixed histology types & 37 & 26 \\
\hline
\end{tabular}


negative lymph nodes retrieved, irrespective of the nomenclature used by surgeons in identifying the location of lymph nodes within a resected specimen (eg, gastric lesser and greater curvatures, and the paragastric, paracardiac, and paraesophageal areas, and so on). The pathological nodal (pN) stage was assigned on the basis of the number of involved nodes. Celiac axis nodes were retrieved by some surgeons in the celiac, left gastric, hepatic, and splenic arterial regions. As defined by both the AJCC 6th and AJCC 7th staging systems, these nodes were classified as regional for cancer of the stomach. However, for cancer of the gastroesophageal junction and proximal stomach ('cardia'), they have been variably designated as regional by some ${ }^{9}$ or as distant by other investigators. ${ }^{7}$ In this study, we defined any metastasis identified in these nodes as distant metastasis, because gastric cardiac carcinoma involving the esophagus is considered as gastroesophageal junction cancer and believed to be esophageal in origin. ${ }^{7,8}$ Limited lymphadenectomy was performed in the paraesophageal region of the lower mediastinum in 10 patients, and no involved nodes were identified. Tumor deposits in distant organs such as the liver $(n=6)$ were also staged as pathological metastasis (1) (pM1); otherwise, the case was assigned as pM0, as defined by the AJCC 7th system.

In this comparison study, we first used the rules for gastric cancer defined by the AJCC 7th system to stage and compare the pathological staging results between the AJCC 6th and AJCC 7th stage grouping schemes on these tumors. Then, we applied the rules for esophageal cancer defined by the AJCC 7th to stage the same cases and compare pathological TNM stages with those generated by the gastric cancer staging scheme.

\section{Statistical Analysis}

Patient demographic and tumor pathology characteristics were described, using tables for categorical data, median and standard deviation (s.d.), and ranges for continuous variables. The data were presented as median \pm s.d. The number of months after surgery was calculated from the month of surgical resection to the month of last follow-up interview in May 2010 or the death of a patient from any cause. The Kaplan-Meier survival curves were estimated and tested with the log-rank test for statistical significance. We used the Statistical Package for Social Sciences (SPSS, version 13.0, Chicago, IL, USA) statistical software to compare pT, $\mathrm{pN}$, and overall stages between the two cancer staging systems. The difference with the $P$-value of $<0.05$ was considered statistically significant.

\section{Results}

The patient demographic features were shown in Table 1 and were similar to those reported previously in Chinese patients from China, Taiwan and in Japanese patients. ${ }^{2,10}$ The median number of months of patient follow-up was $29( \pm 17$; range, 8-70). By the end of follow-up, 58 of $142(41 \%)$ patients died, and the remainders were censored. Abdominal proximal or total gastrectomies were carried out in over $90 \%$ of cases. The median tumor size was $5.0 \mathrm{~cm}( \pm 2$; range, $0.5-12)$. Although the majority $(79 \%)$ of tumors were adenocarcinomas (Table 1), a variety of uncommon tumor histological types were noted (Table 1), as described previously. ${ }^{11-13}$

\section{pT Staging}

According to the AJCC 7th for gastric cancer, the vast majorities (over $87 \%$ ) of tumors invaded into the subserosa and were staged pT3. There was only one case showing tumor extending into the adjacent splenic capsule and focally into the spleen, which was staged as pT4b. However, the differences in all categories of pT stages were not statistically significant between the AJCC 7th and AJCC 6th systems (Table 2).

\section{pN Staging}

A total of 2960 lymph nodes (median: 21 per case; range, 1-66) in 142 cases were retrieved and evaluated histologically. There were 100 cases $(70 \%)$ with the number of total nodes greater than 15 per case. Both the median number of total nodes per case and the number of cases with over 15 nodes were similar to those reported in Japan. ${ }^{14}$ Metastasis was found in $825(28 \%)$ nodes. There were 106 $(75 \%)$ cases with a median number of $6(5.7 \pm 6$; range, 1-25) positive nodes per case.

For the $\mathrm{pN}$ stage as a gastric cancer, both the AJCC 7th and AJCC 6th systems use the number, not the site, of involved lymph nodes as the prognostic discriminator. The new $\mathrm{pN}$ system defines $\mathrm{pN} 1$ with 1-2 positive nodes, rather than 1-6 nodes required by the old system, and the new pN2 with 3-6 positive nodes. If the number of involved nodes is over 7 or 16 , the new system designates them as pN3A or pN3B, respectively. As a result, over $57 \%$ of cases staged at old $\mathrm{pN} 1$ and $85 \%$ of old pN2 were upstaged into the new pN2 and pN3A stages, respectively. In addition, over $93 \%$ of old pN3 cases were upstaged to the new pN3B (Figure 1). The cases staged at new pN3 were universally associated with advanced tumor stages, and accounted for $100 \%$ of new stage pIIIB and $83 \%$ of stage pIV cases. In addition, the 5-year survival rate for patients staged at $\mathrm{pN} 3 \mathrm{~B}$ was worse than that at $\mathrm{pN} 3 \mathrm{~A}, \mathrm{pM} 1$, and even new overall stage pIV (Table 2). However, the differences between the old and new $\mathrm{pN}$ systems in predicting patient survival were significant for $\mathrm{pN} 2$ $(P=0.0004)$.

\section{pM Staging}

Both the AJCC 7th and AJCC 6th systems define pM1 as distant metastasis. For carcinoma of the gastroesophageal junction and gastric cardiac 
Table 2 Comparison of pathological TNM stage between the 7th and 6th AJCC staging systems

\begin{tabular}{|c|c|c|c|c|c|c|c|}
\hline \multirow[t]{2}{*}{ pTNM } & \multirow[t]{2}{*}{ Stage } & \multirow[t]{2}{*}{ Number } & \multirow[t]{2}{*}{$M A S$} & \multicolumn{3}{|c|}{ Survival rate (\%) } & \multirow[t]{2}{*}{ P-value } \\
\hline & & & & 1 year & 3 year & 5 year & \\
\hline \multirow[t]{5}{*}{ pT (6th edition) } & 1 & 4 & 43 & 100.00 & 75.00 & 75.00 & \\
\hline & $2 \mathrm{~A}$ & 12 & 27 & 83.33 & 83.33 & 75.35 & \\
\hline & $2 \mathrm{~B}$ & 2 & 37 & 100.00 & 50.00 & 50.00 & \\
\hline & 3 & 123 & 29 & 86.99 & 73.98 & 58.54 & \\
\hline & 4 & 1 & 18 & 100.00 & 0.00 & 0.00 & 0.3163 \\
\hline \multirow[t]{6}{*}{ pT (7th edition) } & $1 \mathrm{~A}$ & 2 & 38 & 100.00 & 50.00 & 50.00 & \\
\hline & 1B & 2 & 47 & 100.00 & 100.00 & 100.00 & \\
\hline & 2 & 12 & 27 & 83.33 & 72.92 & 54.69 & \\
\hline & 3 & 125 & 29 & 87.04 & 59.83 & 29.23 & \\
\hline & $4 \mathrm{~A}$ & 0 & 0 & - & - & - & \\
\hline & $4 \mathrm{~B}$ & 1 & 18 & 100.00 & 0.00 & 0.00 & 0.3276 \\
\hline \multirow[t]{5}{*}{$\mathrm{pN}$ (6th edition) } & 0 & 36 & 35 & 94.44 & 72.02 & 53.50 & \\
\hline & 1 & 51 & 30 & 87.96 & 71.30 & 56.44 & \\
\hline & 2 & 40 & 26 & 84.62 & 54.08 & 8.56 & \\
\hline & 3 & 15 & 20 & 74.48 & 16.67 & 0.00 & \\
\hline & CAN(+) & 10 & 15 & 80.00 & 16.67 & 16.67 & 0.0000 \\
\hline \multirow{5}{*}{ pN (7th edition) } & 0 & 36 & 35 & 94.44 & 72.02 & 53.50 & \\
\hline & 1 & 22 & 32 & 91.30 & 70.06 & 70.06 & \\
\hline & 2 & 35 & 30 & 85.32 & 64.66 & 15.91 & \\
\hline & $3 \mathrm{~A}$ & 35 & 25 & 85.29 & 57.43 & 17.36 & \\
\hline & $3 \mathrm{~B}$ & 14 & 15 & 70.71 & 10.31 & 10.31 & 0.0002 \\
\hline \multirow{2}{*}{ pM (6th edition) } & $\mathrm{x}$ & 130 & 30 & 85.75 & 61.60 & 43.95 & \\
\hline & 1 & 13 & 16 & 64.96 & 12.96 & 12.96 & 0.0037 \\
\hline \multirow[t]{2}{*}{ pM (7th edition) } & 0 & 130 & 30 & 88.30 & 64.46 & 32.49 & \\
\hline & 1 & 13 & 16 & 75.00 & 13.13 & 13.13 & 0.0003 \\
\hline \multirow[t]{6}{*}{ pTNM (6th edition) } & IA & 4 & 43 & 100.00 & 75.00 & 75.00 & \\
\hline & IB & 8 & 34 & 100.00 & 100.00 & 87.50 & \\
\hline & II & 27 & 33 & 85.19 & 60.68 & 53.10 & \\
\hline & IIIA & 49 & 32 & 91.65 & 71.65 & 52.95 & \\
\hline & IIIB & 30 & 28 & 86.67 & 63.71 & 11.38 & \\
\hline & IV & 24 & 18 & 74.51 & 17.76 & 17.76 & 0.0000 \\
\hline \multirow[t]{8}{*}{ pTNM (7th edition) } & IA & 4 & 43 & 100.00 & 75.00 & 75.00 & \\
\hline & IB & 7 & 30 & 100.00 & 100.00 & 100.00 & \\
\hline & IIA & 26 & 34 & 88.46 & 63.01 & 42.01 & \\
\hline & IIB & 26 & 32 & 84.62 & 66.75 & 60.68 & \\
\hline & IIIA & 29 & 31 & 93.10 & 72.57 & 19.79 & \\
\hline & IIIB & 29 & 27 & 86.21 & 62.91 & 15.73 & \\
\hline & IIIC & 9 & 18 & 76.19 & 15.24 & 15.24 & \\
\hline & IV & 13 & 16 & 75.00 & 13.13 & 13.13 & 0.0009 \\
\hline
\end{tabular}

Abbreviations: CAN+: positive celiax axis nodes; MAS: months after surgery.

carcinomas involving the esophagus, celiac axis nodal metastasis is considered distant metastasis by some investigators. ${ }^{7}$ Lymphadenectomy in the celiac axis region was carried out in 44 (31\%) cases, and celiac axis nodal metastasis was detected in 12 $(27 \%)$ cases. We found that patients with positive celiac axis nodes had 3- and 5-year survival rates worse than those staged pIIIB and pIIIA, respectively (Table 2), and slightly better than those staged pM1 and pIV (Table 2, Figure 2). The results justified their being classified as distant metastasis. In this study, there were 6 cases with hepatic and 10 cases with celiac axis nodal metastasis, including 6 in the left gastric arterial, 3 in the hepatic arterial, and 1 in the splenic arterial regions. Because of overlapping in 3 cases with both liver metastasis and celiac axis nodal involvement, the total number of cases staged as pM1 by either the old or new pM system was 13 (Table 2).

\section{Overall Pathological Staging}

As shown in Tables 2 and 3, the new AJCC 7th system is much more detailed, with subcategories of overall stages pII and pIII. The cases staged at pIIIA by the AJCC 6th system had a 3-year survival rate better than those at pII (Table 2, Figure 3). This error was partially corrected by the AJCC 7th system. All old pIII cases were spread among the new subcategories of pIIB, pIIIA, and pIIIB in the AJCC 7th system (Tables 2 and 3). In contrast, stage pIV tumors by the AJCC 6th system were spread among new stages PIIIB, pIIIC, and pIV by the AJCC 7th system and were visually dramatically separated 

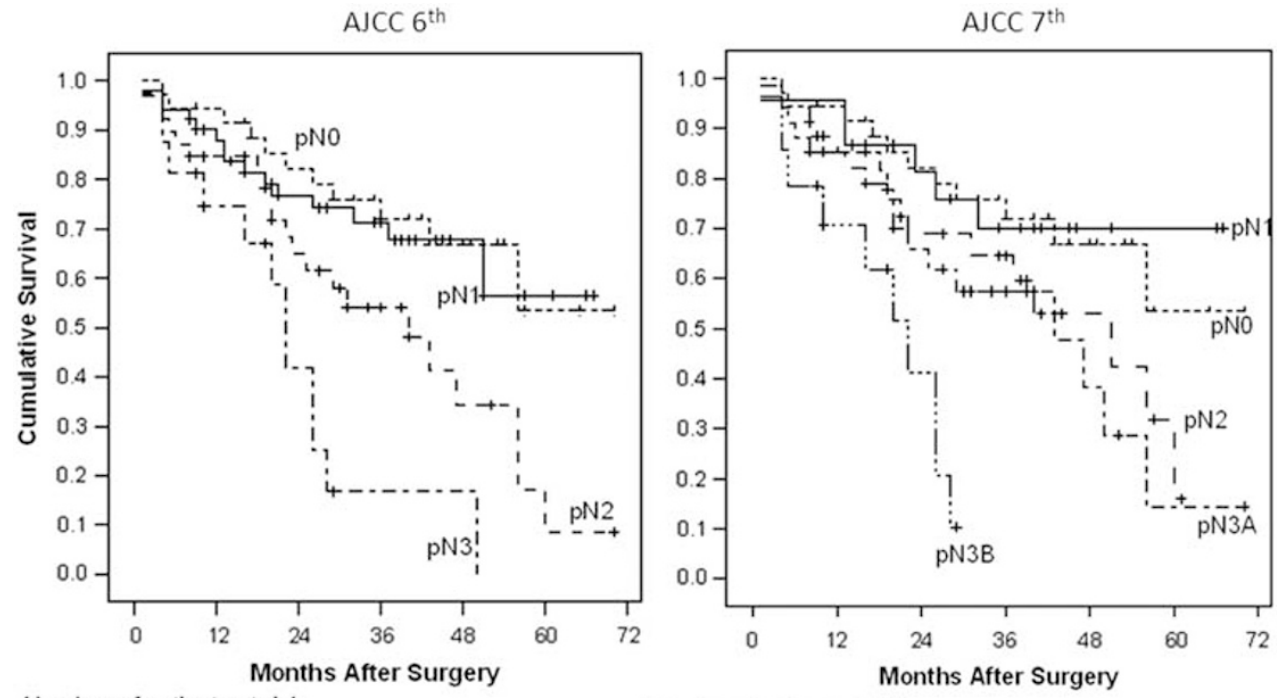

Number of patients at risk

$\begin{array}{llcccc}\text { pN0: } 36 & 34 & 30 & 27 & 26 & 25 \\ \text { pN1: } 51 & 45 & 40 & 38 & 37 & 37 \\ \text { pN2: } 39 & 32 & 26 & 22 & 21 & 19 \\ \text { pN3: } 16 & 12 & 8 & 5 & 5 & 4\end{array}$

Number of patients at risk

$\begin{array}{ccccccc}\text { pN0: } & 36 & 34 & 30 & 27 & 26 & 25 \\ \text { pN1: } & 23 & 21 & 19 & 17 & 17 & 17 \\ \text { pN2: } & 35 & 30 & 26 & 25 & 22 & 19 \\ \text { pN3A: } & 34 & 28 & 23 & 20 & 20 & 19 \\ \text { pN3B: } & 14 & 9 & 7 & 6 & 4 & 4\end{array}$

Figure 1 Staged as gastric cancer, the $\mathrm{pN}$ stage of gastric cardiac carcinoma involving the esophagus was better stratified by the AJCC 7th than the 6th system. Crossed marks represent censored cases.

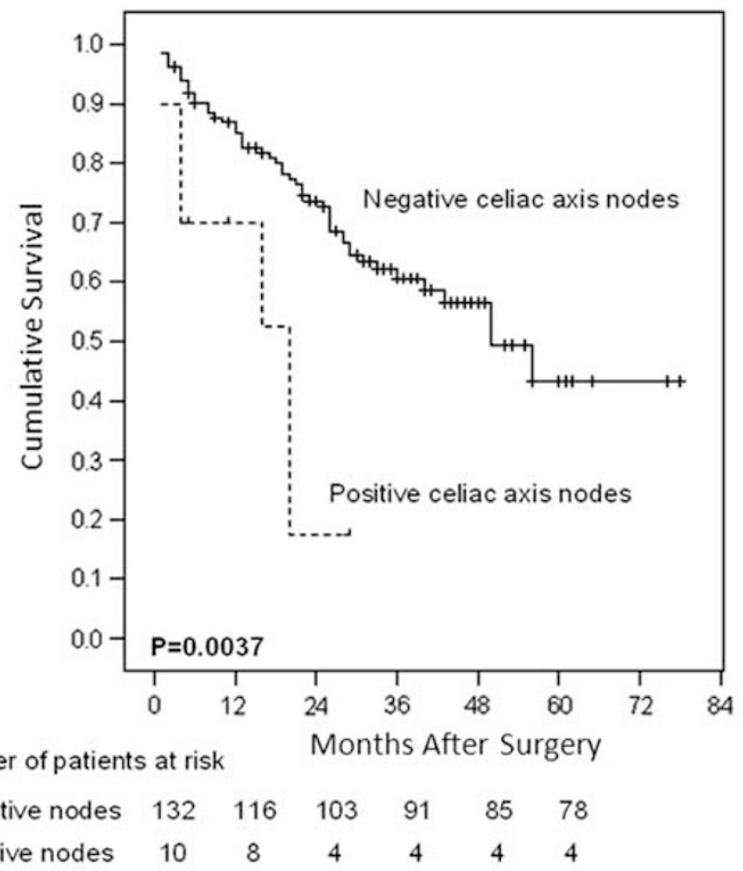

Figure 2 The patients with gastric cardiac carcinoma involving the esophagus and cancer metastasis in the celiac axis lymph nodes had significantly worse prognosis than those without. Crossed marks represent censored cases.

(Figure 3). However, because of the absence of instruction in the AJCC 7th system on how to designate cases with $\mathrm{pN} 3 \mathrm{~A}$ and $\mathrm{pN} 3 \mathrm{~B}$ into the new overall staging system, we grouped these pN3A and pN3B cases together into one pN3 stage for overall
Table 3 Joint overall pathological stage

\begin{tabular}{ccccccccccc}
\hline & & \multicolumn{7}{c}{ AJCC 7th edition } \\
\cline { 3 - 10 } & & Stage & 1A & IB & IIA & IIB & IIIA & IIIB & IIIC & IV \\
\hline \multirow{2}{*}{ AJCC 6th edition } & IA & 4 & & & & & & & \\
& IB & & 7 & 1 & & & & & \\
& II & & & 25 & 1 & & & & \\
& IIIA & & & & 25 & 24 & & & \\
& IIIB & & & & & 5 & 24 & 1 & \\
IV & & & & & 4 & 9 & 13
\end{tabular}

staging assignment. The AJCC 7th system restricts designation of stage pIV to cases with distant metastasis only. As such, 13 cases in our cohort were qualified for pIV (Table 2). Apparently, the AJCC 7th system greatly helps to stratify advanced gastric cardiac carcinomas involving the esophagus more evenly into pIIB, pIIIA, pIIIB, and pIIIC subcategories, so that these advanced tumors were not skewed toward pIIIA, as in the AJCC 6th system (Tables 2 and 3, Figure 3). We found that the patients staged at new pT3 and pN3B $(n=14)$ had the 5-year survival rate worse than those at $\mathrm{pM} 1$ and $\mathrm{pIV}$ (Table 2). Therefore, for better prediction of patient survival, we propose designating gastric cardiac carcinomas involving the esophagus staged at pT3 and $\mathrm{pN} 3 \mathrm{~B}$ as pIV in the future edition of the AJCC cancer staging system. Despite the combined efforts, the survival curves of patients with gastric cardiac carcinomas involving the esophagus staged at pIIA, pIIB, pIIIA, and pIIIB remained crossed (Figure 3 ), indicating 

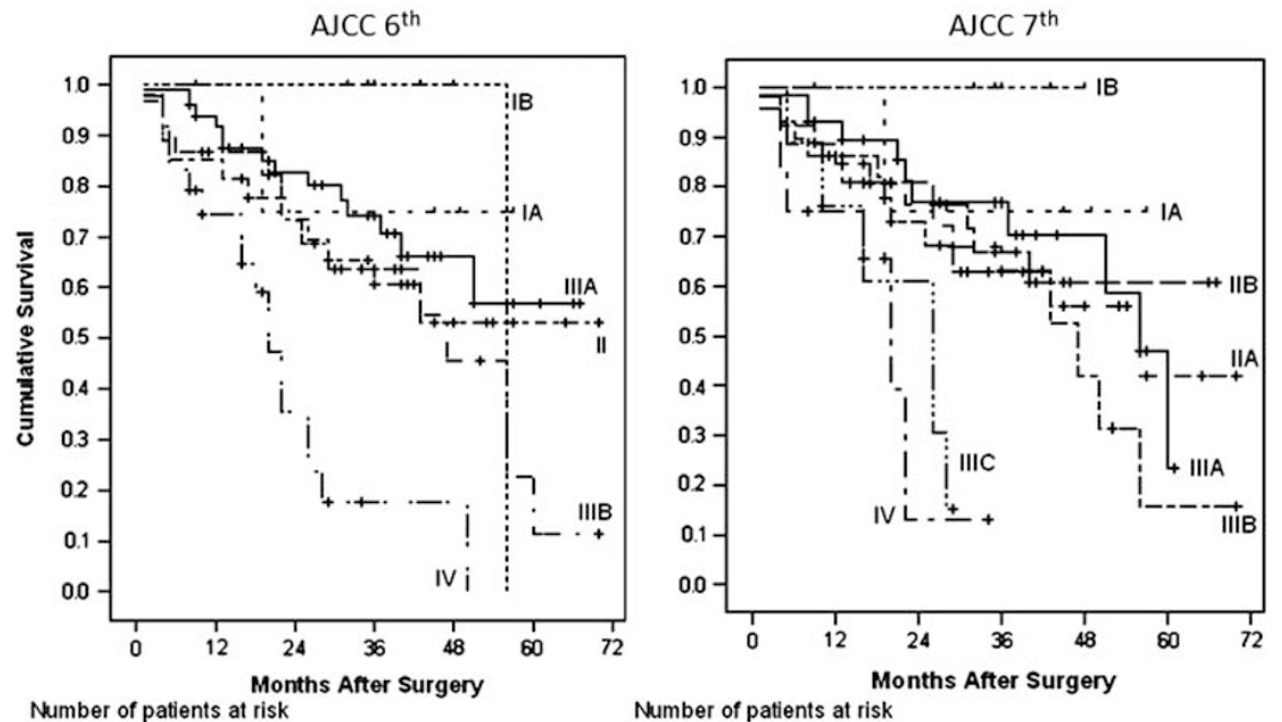

$\begin{array}{rrrrrrr}\text { IA: } & 4 & 4 & 3 & 3 & 3 & 3 \\ \text { IB: } & 8 & 8 & 8 & 8 & 8 & 7 \\ \text { II: } & 27 & 23 & 20 & 17 & 16 & 16 \\ \text { IIIA: } & 49 & 45 & 41 & 38 & 36 & 35 \\ \text { IIIB: } & 30 & 26 & 22 & 21 & 19 & 16 \\ \text { IV: } & 24 & 18 & 11 & 8 & 8 & 7\end{array}$

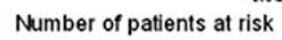

$\begin{array}{ccccccc}\text { IA: } & 4 & 4 & 4 & 3 & 3 & 3 \\ \text { IB: } & 7 & 7 & 7 & 7 & 7 & 7 \\ \text { IIA: } & 26 & 22 & 21 & 17 & 16 & 16 \\ \text { IIB: } & 26 & 22 & 21 & 17 & 17 & 17 \\ \text { IIIA: } & 29 & 27 & 23 & 23 & 22 & 19 \\ \text { IIIB: } & 29 & 25 & 22 & 20 & 18 & 16 \\ \text { IIIC: } & 9 & 7 & 6 & 3 & 3 & 3 \\ \text { IV: } & 13 & 8 & 4 & 4 & 4 & 4\end{array}$

Figure 3 Staged as gastric cancer, gastric cardiac carcinomas involving the esophagus were better stratified in the overall stages by the AJCC 7th than 6th system. Crossed marks represent censored cases.

the existence of substantial room for improvement of the AJCC cancer staging system on this fatal cancer.

\section{Staging with the Esophageal Cancer Scheme}

As shown in Figure 4, the overall stage of this group of cancer by the esophageal cancer staging rules was not significantly better stratified than that generated with the gastric cancer staging system. The KaplanMeier survival curves for patients with advanced cancers staged at pIIIA were erroneously better than those staged at pIIB and pIA. In addition, the curves of pIIB and pIIIB were also crossed, indicating the existence of heterogeneous patient populations within these categories.

\section{Discussion}

To our knowledge, this is the first study attempting to independently validate the improved predictive value of the new AJCC 7th system for surgical therapy outcomes of patients with gastric cardiac carcinomas involving the esophagus. In this study, we used strict patient selection criteria to avoid confounding factors such as neoadjuvant therapyassociated changes in tumor gross and histological characteristics, and limited our study to cases with tumor epicenters within $5 \mathrm{~cm}$ below the gastroesophageal junction. Therefore, these tumors belonged to carcinomas of the gastroesophageal junction. ${ }^{8,15}$
In gastric cancer, lymph node metastasis has a significant role in predicting survival, and is more important as a prognostic indicator than the pT stage. This study confirmed that the presence of any lymph node metastasis was consistently associated with a dismal prognosis. ${ }^{7}$ We also showed that the new $\mathrm{pN}$ system was superior to the previous version, as old $\mathrm{pN} 2$ cases can be better substratified into $\mathrm{pN} 2$ and pN3A stages. Therefore, the current overall stage pII tumors were better distinguished from stage pIII cancers. Interestingly, all patients staged at pN3B died early and had worse 3- and 5year survival rates than those staged at pM1 and even at pIV. We propose that in future modifications of the AJCC 7th system, consideration should be made for combining pT3 and pN3B cases into the overall stage pIV. Obviously, this proposal requires confirmation and validation in larger prospective trials.

Lymphatic spread from gastric cardiac carcinomas involving the esophagus was reported primarily in abdominal sites, in the decreasing order of frequency of the paracardia, lesser and greater curvatures, celiac axis, retropancreas, and lower mediastinum. ${ }^{16,17}$ Neither the old nor new $\mathrm{pN}$ staging systems consider the prognostic significance of specific sites of lymph node metastasis. This may be due to the variable frequency of involvement of these nodal sites, the complexity and subjectivity of nodal stations, surgeons' varying experience in nodal 


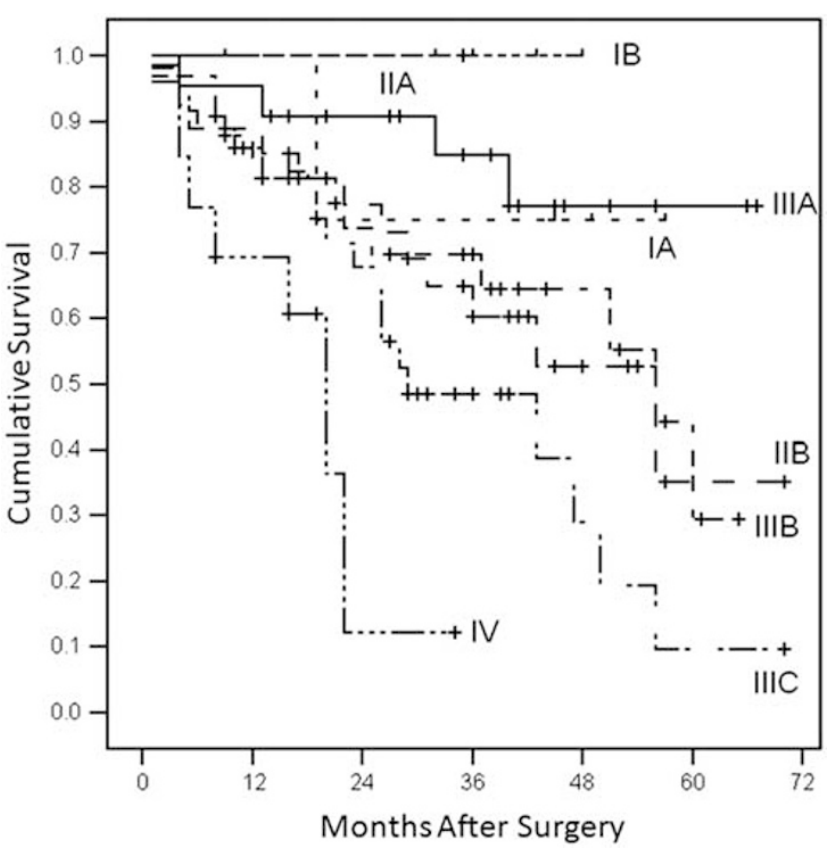

Figure 4 With the guidelines for esophageal cancer staging of the AJCC 7th system, the survival curves for patients with advanced cancers staged at pIIIA were erroneously much better than those staged at pIA and pIIB. The curves of pIIB and pIIIB were also crossed. Crossed marks represent censored cases.

dissection, and an inconsistent relationship of nodal disease to previously reported outcomes. ${ }^{18-20}$ In our series, the nodes in the retropancreatic, paraaortic, and paraesophageal regions were negative for malignancy, and most cases showed nodal metastasis, in the decreasing order of frequency, at the paracardial, the lesser and greater curvatures, and the celiac axis regions. Celiac axis nodal metastasis was present in $27 \%$ of cases, a rate similar to that reported in the literature. ${ }^{16,21}$

The presence of nodal metastasis in this celiac axis second-tier nodal system indicates cancer progression. As such, the 5-year survival rate in our patients was only about $18 \%$, which was slightly better than those staged at pM1, pIIIC, and pIV. This rate was similar to that reported in Japanese patients ${ }^{22}$ and slightly better than that described in a similar study from Taiwan, in which no long-term survival was observed in patients with celiac nodal diseases. ${ }^{2}$ Recently, Schomas et $a l^{23}$ systematically investigated the prognostic significance of celiac axis nodal involvement in patients with gastroesophageal junction cancer and found that the survival rates of those with celiac nodal metastasis were comparable with those of $\mathrm{pN} 1$, and similar to patients with stage pIII disease. However, over $52 \%$ of their patients had cancers within the distal esophagus above the gastroesophageal junction; therefore, their data may not be comparable with ours. It is apparent that the prognostic importance of celiac nodal metastasis in patients with gastric cardiac carcinomas involving the esophagus cannot be ignored and, in fact, the site of involved nodes, such as celiac axis nodal metastasis, is as important as the number of positive nodes for staging and prognostic prediction. ${ }^{14,16}$ Because of the small sample size of the present cohort, we cannot absolutely conclude that celiac axis nodal disease is the most prognostically crucial factor. Nevertheless, because a dismal prognosis is strongly associated with the involvement of celiac axis nodes, adequate preoperative staging, which can be achieved by combining abdominal CT and ultrasound techniques, is critically important in selecting patients for optimal therapy. ${ }^{9}$ Total gastrectomy with en-bloc resection of advanced gastric cardiac carcinomas involving the esophagus along with the surrounding lymph nodes in the celiac axis region has been advocated to improve patient survival. ${ }^{21,23}$

We found herein that the AJCC 7th system was not as good for prognostic stratification of patients with gastric cardiac carcinomas involving the esophagus when they were grouped as esophageal cancers, compared with when staged as gastric cancers. ${ }^{8}$ These results will certainly fuel the ongoing controversy debate about the pathogenesis, origin, and classification of gastroesophageal junction cancers and gastric cardiac carcinomas involving the esophagus. Although gastric cardiac carcinoma involving the esophagus is centered in the proximal stomach, some workers consider it esophageal in origin on the basis of epidemiological, molecular, clinicopathological, and survival features that are similar to those of distal esophageal cancer above the gastroesophageal junction. ${ }^{24-26}$ Consequently, these authors have advocated staging and treating these tumors as esophageal cancer. ${ }^{25,27}$

Whether or not the patient population under study influenced our results is worth investigating, gastroesophageal junction cancer is common in China, and almost all tumors in this population are centered in the proximal stomach, in contrast to the vanishingly rare distal esophageal adenocarcinoma above the gastroesophageal junction..$^{2,11,28-30}$ In a recent study from a high-volume medical center in China, Taiwan, the investigators did not find a single case of such cancer over a 20-year period. ${ }^{2}$ In our most recent comparison study on gastroesophageal junction cancer, we discovered different clinicopathological features of this cancer between Chinese patients in Nanjing, China and American Caucasian patients in Boston, the United States. ${ }^{11}$ Compared with American patients, Chinese patients were younger at cancer diagnosis and showed a higher female-male ratio. Their tumors were larger in size, more advanced in the pathology stage, and more frequently associated with chronic Helicobacter pylori gastritis and dysplasia. In contrast, esophageal columnar intestinal metaplasia and dysplasia are the common precursors to distal esophageal cancer above the gastroesophageal junction in American patients, but 
not so in Chinese. Importantly, the outcome of Chinese patients with stage pIII cancers was significantly better than that of American patients. ${ }^{31}$ Taken together, the data from our and others studies indicate that gastric cardiac carcinomas involving the esophagus should be classified and staged as gastric cancer, rather than esophageal. ${ }^{2,11,29,31}$

There are several major limitations of this study. First, this is a retrospective study, and most surgical procedures were carried out by different surgeons using nonstandardized surgical approaches. This caused inconsistent lymphadenectomy and collection of resection specimens. Most likely, the extent of nodal involvement was underestimated. The lack of information of nodal status at specific remote sites in most cases also made impossible the investigation of nodal stage migration. ${ }^{32}$ Second, over $55 \%$ of our cases were staged at pIII and pIV and a few cases with the entire gastroesophageal junction involved by cancer. This might have resulted in underassigning distal esophageal cancers above the gastroesophageal junction. However, all our patients were Chinese, in whom Barrett esophagus is extremely rare,${ }^{11}$ and the risk that distal esophageal cancer might have been misclassified is very low. Third, most of our cases were advanced tumors, and only a few were staged at pI and pII. Thus, we were unable to evaluate the AJCC 7th system fully. To address these limitations, we have initiated a prospective investigation with a much larger patient sample size to overcome these shortcomings.

\section{Conclusions}

Compared with the AJCC 6th system, the new AJCC 7th system better separated stage pIII gastric cardiac carcinomas involving the esophagus and better predicted patient survival when staged as gastric cancers. However, the AJCC 7th system provided insufficient guidance for staging advanced gastric tumors, such as pT3 with pN3B. In the latter, we propose a minor modification to the AJCC 7th system by combining pT3 with pN3B as pIV. For pN staging, the prognostic significance of the celiac axis nodal metastasis in patients with gastric cardiac carcinomas involving the esophagus should be taken seriously and further investigated. In addition, we provided the evidence that, at least in the Chinese population, gastric cardiac carcinoma involving the esophagus is better staged and stratified by the rules for gastric, not esophageal, cancer.

\section{Acknowledgements}

This project was financed partially by a grant from the Science and Technology Development Project of the Nanjing City (No. 200601050) and a special grant from the Nanjing Drum Tower Hospital, Nanjing, China.

\section{Disclosure/conflict of interest}

The author declares no conflict of interest.

\section{References}

1 American Joint Committee on Cancer. Digestive system. AJCC Cancer Staging Manual 6th edn. Springer: New York, 2002 pp 91-103.

2 Fang WL, Wu CW, Chen JH, et al. Esophagogastric junction adenocarcinoma according to siewert classification in Taiwan. Ann Surg Oncol 2009;16: 3237-3244.

3 de Manzoni G, Pedrazzani C, Pasini F, et al. Results of surgical treatment of adenocarcinoma of the gastric cardia. Ann Thorac Surg 2002;73:1035-1040.

4 Mariette C, Castel B, Toursel H, et al. Surgical management of and long-term survival after adenocarcinoma of the cardia. Br J Surg 2002;89:1156-1163.

5 DeMeester SR. Adenocarcinoma of the esophagus and cardia: a review of the disease and its treatment. Ann Surg Oncol 2006;13:12-20.

6 Lagarde SM, ten Kate FJ, Reitsma JB, et al. Prognostic factors in adenocarcinoma of the esophagus or gastroesophageal junction. J Clin Oncol 2006;24: 4347-4355.

7 Rizk NP, Venkatraman E, Bains MS, et al. American joint committee on cancer staging system does not accurately predict survival in patients receiving multimodality therapy for esophageal adenocarcinoma. J Clin Oncol 2007;25:507-512.

8 American Joint Committee on Cancer. Chapter 10. Esophagus and esophagogastric junction. In: AJCC Cancer Staging Manual 7th edn. Springer: New York, NY, 2009, pp 129-144.

9 van Vliet EP, Steyerberg EW, Eijkemans MJ, et al. Detection of distant metastases in patients with oesophageal or gastric cardia cancer: a diagnostic decision analysis. Br J Cancer 2007;97:868-876.

10 Kusano C, Gotoda T, Khor CJ, et al. Changing trends in the proportion of adenocarcinoma of the esophagogastric junction in a large tertiary referral center in Japan. J Gastroenterol Hepatol 2008;23: 1662-1665.

11 Huang Q, Fan XS, Agoston AT, et al. Gastroesophageal junctional carcinomas in Chinese patients show distinct clinicopathologic features [abstract]. Mod Pathol 2009;22(Suppl.1) Abstract 133A.

12 Huang Q, Zhang LH. The histopathologic spectrum of carcinomas involving the gastroesophageal junction in the Chinese. Int J Surg Pathol 2007;15:38-42.

13 Siewert JR, Feith M. Adenocarcinoma of the esophagogastric junction: competition between Barrett and gastric cancer. J Am Coll Surg 2007;205(4 Suppl): S49-S53.

14 Hayashi H, Ochiai T, Suzuki T, et al. Superiority of a new UICC-TNM staging system for gastric carcinoma. Surgery 2000;127:129-135.

15 Siewert JR, Stein HJ. Classification of adenocarcinoma of the oesophagogastric junction. Br J Surg 1998;85: 1457-1459.

16 de Manzoni G, Verlato G, Guglielmi A, et al. Classification of lymph node metastases from carcinoma of the stomach: comparison of the old (1987) and new (1997) TNM systems. World J Surg 1999;23: $664-669$. 
17 von Rahden BH, Feith M, Stein HJ. Carcinoma of the cardia: classification as esophageal or gastric cancer? Int J Colorectal Dis 2005;20:89-93.

18 Hulscher JB, Buskens CJ, Bergman JJ, et al. Positive peritruncal nodes for esophageal carcinoma: not always a dismal prognosis. Dig Surg 2001;18: 98-101.

19 Dresner SM, Lamb PJ, Bennett MK, et al. The pattern of metastatic lymph node dissemination from adenocarcinoma of the esophagogastric junction. Surgery 2001;129:103-109.

20 de Manzoni G, Pedrazzani C, Verlato G, et al. Comparison of old and new TNM systems for nodal staging in adenocarcinoma of the gastro-oesophageal junction. Br J Surg 2004;91:296-303.

21 Roviello F, Marrelli D, Morgagni P, et al. Italian research group for gastric cancer. survival benefit of extended D2 lymphadenectomy in gastric cancer with involvement of second level lymph nodes: a longitudinal multicenter study. Ann Surg Oncol 2002;9:894-900.

22 Nunobe S, Ohyama S, Sonoo H, et al. Benefit of mediastinal and para-aortic lymph-node dissection for advanced gastric cancer with esophageal invasion. J Surg Oncol 2008;97:392-395.

23 Schomas DA, Quevedo JF, Donahue JM, et al. The prognostic importance of pathologically involved celiac node metastases in node-positive patients with carcinoma of the distal esophagus or gastroesophageal junction: a surgical series from the Mayo Clinic. Dis Esophagus 2009;23:232-239.

24 Wijnhoven BP, Siersema PD, Hop WC, et al. Adenocarcinomas of the distal oesophagus and gastric cardia are one clinical entity. Rotterdam Oesophageal Tumour Study Group. Br J Surg 1999;86:529-535.

25 Demeester SR. Epidemiology and biology of esophageal cancer. Gastrointest Cancer Res 2009;3(2 Suppl): S2-S5.

26 Whitson BA, Groth SS, Li Z, et al. Survival of patients with distal esophageal and gastric cardia tumors: a population-based analysis of gastroesophageal junction carcinomas. Thorac Cardiovasc Surg 2009;139: 43-48.

27 Marsman WA, Tytgat GN, ten Kate FJ, et al. Differences and similarities of adenocarcinomas of the esophagus and esophagogastric junction. J Surg Oncol 2005;92: 160-168.

28 Law SY, Fok M, Cheng SW, et al. A comparison of outcome after resection for squamous cell carcinomas and adenocarcinomas of the esophagus and cardia. Surg Gynecol Obstet 1992;175:107-112.

29 Wang LD, Zheng S, Zheng ZY, et al. Primary adenocarcinomas of lower esophagus, esophagogastric junction and gastric cardia: in special reference to China. World J Gastroenterol 2003;9:1156-1164.

30 Kamangar F, Qiao YL, Blaser MJ, et al. Helicobacter pylori and oesophageal and gastric cancers in a prospective study in China. Br J Cancer 2007;96:172-176.

31 Fan XS, Feng AN, Lauwers GY, et al. Esophageal columnar metaplasia is common in the distal esophagus of Chinese patients. Gastroenterology 2010; 138(Suppl 1) Abstract S-758.

32 de Manzoni G, Verlato G, Roviello F, et al. The new TNM classification of lymph node metastasis minimises stage migration problems in gastric cancer patients. Br J Cancer 2002;87:171-174. 